\title{
Cell Shrinkage, Cytoskeletal Pathologies, and Neurodegeneration: Myelin Sheath Formation and Remodeling
}

\section{Rajiv Kumar*}

*Correspondence: Rajiv Kumar

Address: NIET, National Institute of Medical Science, India

e-mail $\bowtie$ : chemistry_rajiv@hotmail.com

Received: 23 March 2021; Accepted: 26 March 2021

Copyright: (C) 2021 Rajiv K. This is an open-access article distributed under the terms of the Creative Commons Attribution License, which permits unrestricted use, distribution, and reproduction in any medium, provided that the original work is properly cited.

\section{Opinion}

Cell shrinkage is a biological process and when it started naturally or by any means, it indicates programmed cell death. A proper understanding of cell volume regulation, and routes involved in the movement of ions at the activation spot of apoptosis in the cell, can disclose the cell shrinkage process (Russo et al., 2013). Focused research on the concentration of potassium and sodium ions further leads to disclose the concerned process in maintaining the normal physiological intracellular concentration (Alisafaei et al., 2019) (Fig. 1).

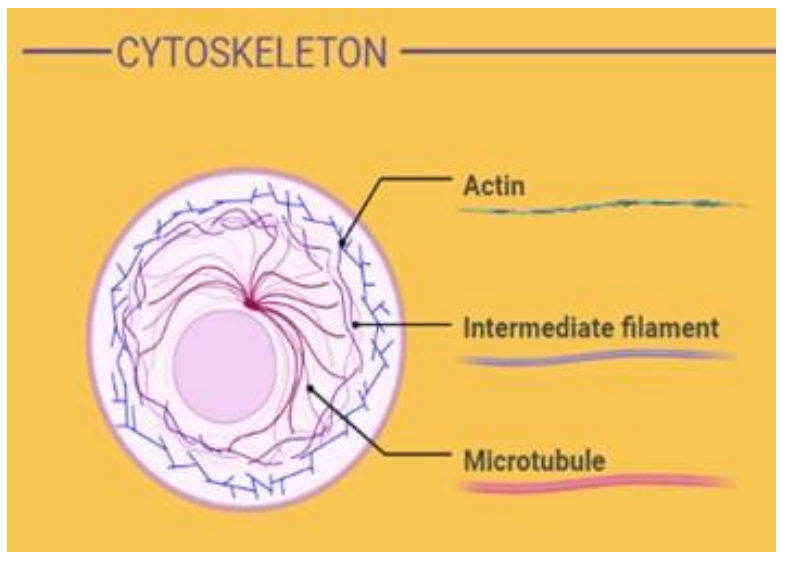

Figure 1: Illustration of Cell shrinkage, disorders cytoskeletal, myelin sheath formation and remodeling. "Adapted and created with permission from [biorender.com] and acknowledged as per instructions

Furthermore, during the initiation of apoptosis, the role of the said ions has more concerns with the loss of cell volume. The authors explored the concepts of cell volume regulation whenever apoptosis happened, interlinked routes of ion movement, and disclosing the activation routes at the time of cell death (Elmore, 2007). It is a well-known fact that the processes of cell shrinkage and programmed cell death are transpired altogether. Simultaneously, the concentration of potassium also compels the 
participation of death cascades in the aforementioned biological routes and paths. Accordingly, intracellular potassium also initiates a cellular process to block apoptosis. All in all, the understanding of ion movements and fluxes of intracellular ions $(\mathrm{K}, \mathrm{Na}$, and $\mathrm{Cl})$ divulge the features that instigated the cell death activation and how the cellular swelling is associated with necrosis (Leanza et al., 2013). As discussed features proved that the sodium influx influence the fluctuations in cell size and apoptosis. Various cellular mechanisms (chromatin condensation, internucleosomal DNA degradation, and externalization of phosphatidylserine, caspase activity), which are key biological and chemical transformations, transpired when the routes of cell shrinkage initiate. The aforesaid features were underlined as classical features of apoptosis. Overall, this discussion upkeep that the cell shrinkage and few features of apoptosis are poles apart in many routes and phases.

The discoveries of the cytoskeletal explain the complex features of cell biology and biomedicine. The striated muscle cell cytoskeleton is a multifunctional, and dynamic component of the cell and performs as a platform for inter-and intracellular signaling (Nicolas et al., 2004). It is a complex structure that deals with communications and happens among cell-cell junctions, mitochondria, and the nucleus (Peracchia, 2020). The cell cytoskeleton also links the contractile apparatus for the smooth functioning of sarcomeres and links looked for assemblies to turn the sarcolemma. These biological components and features also assist as a scaffold to uphold the structural integrity and act to design the cell. The cytoskeleton is linking the cell nucleus and the extracellular matrix with each other. These connections govern the communications which occurred via molecules, involved in between, and control gene expression, and cellular processes (Horio et al., 2014). These key features are important ones and help in communications for a better regularization of cellular processes to counter abnormalities that existed in it frequently and caused diseases. Furthermore, the novel discoveries in the research of cytoskeleton and interrelated counter environments, which are responsible for initiating a disease, can defiantly innovate new diagnostic tools and therapeutic remedies in the foreseeable future. Morphological features of the cytoskeleton and functioning style in neurons have a direct concern with the novel cytoskeletal abnormalities. These underlined and highlighted facts can disclose their involvement in neurological diseases.

A better understanding of the pathophysiology of these diseases and the composition of neuron cytoskeletal proteins will disclose the molecular mechanisms underlying neurological diseases (Hoffmann et al., 2009). Such scientific analysis set up the composition of the neuronal cytoskeleton and named actin filaments, microtubules, and neurofilaments as the key component of it. These efforts will enhance the possibilities to understand the functioning style of cytoskeletal proteins for suggesting better treatment. There is no hesitation to state that the understanding of pathophysiological 
mechanisms underlying responsible for neurological disorders will open new avenues for further research and the development of novel remedies required for the treatment. The phenomenon of dysfunction of cytoskeletal mechanisms and constituents disturbed the normal functioning of vesicular biogenesis, vesicle trafficking, organelle working, and synaptic signaling (Muñoz-Lasso et al., 2020). All in all, these disturbances in the smooth functioning initiate mutations, and in the end, several neurodegenerative disorders and other fatal diseases originate in the cellular environment (Jang et al., 2014). The route of dysfunction of cytoskeletal influences a cascade of events (mitochondrial dysfunction and oxidative stress) and finally, initiates DNA damage and alters concerned cellular responses (Kumar et al., 2021). These investigations exposed significant clues that happened in pathophysiology (Bhatti et al., 2017). Therefore, the originated defects disturb the functioning styles and cellular activities of the cytoskeleton. These unnatural happening are responsible for disease initiation and contributing to neurodegeneration. These mechanisms are underlined as the main cause of cell death.

Myelin deals with the flow of the ions and generate a flow of charge and controls the speed of communications in tightly compacted and multi-layered membranes. The understanding of the physiology of myelin remodeling provides the specifics of plasticity, which is a necessity of neuronal networks, applied in communications, if any default observed in it, triggered neurodegeneration (Tonnesen, 2013). The ability to recognize the contacts for better communication is lost and a need is there to expose the physiology of the axons to participate in it. An integrative and multidisciplinary approach is essential to understand how myelin can repair miscarried cellular communication in disease phases. In a normal condition, rapid and efficient communication is persisting within neurons with high speed and can travel in a little time and space. Alternatively, a proper investigation can only be helpful that can open new avenues to have insight into myelination-centered mechanisms governed by neural plasticity. These investigations explore the possibilities to understand the molecular complexity, which deals with these communications, provide an in-depth molecular understanding of the mechanism of axonal interactions and interpretations of these interactions defiantly guide for further research. These cellular activities and communications generate, maintain, and remodel apprehensive myelin sheaths. Here, the author highlights the importance of the biology of myelin, and the connectivity of myelin underlying axons intercellular responses (Chang et al., 2016). More importantly, how any disturbances can initiate innumerable diseases (neuromyelitis optical spectrum disorders, acute disseminated encephalomyelitis, and multiple sclerosis). These interpretations will help to understand the specific interactions that occurred in astrocytes and is another component that transpired at the time of neurodegeneration. In the absence of science about the aforementioned causes and diseases, no pieces of evidence have existed there and the details of events are the only feature that 
was reported in the literature about the molecular phenomenon of remodeling of myelin sheaths and it is a very necessary component to be understood properly (Chapman and Hill, 2020). These interpretations are evident that extensive changes in the mechanism underlying followed all through myelin sheath formation and remodeling. Extensive changes can be done in sheath length and it will helpful in regaining the natural and creative patterns for restoring normal myelination. The axons have abilities and potential that can govern myelin growth, remodeling, and maintenance. The remodeling of the myelin sheath length is the key component that contributes to the proper functioning and repairing to restore the original myelination patterns (Chapman and Hill, 2020). These outputs and findings will help in recovering from neurodegeneration and to treat concerning diseases.

\section{References}

Alisafaei F, Jokhun DS, Shivashankar G V., Shenoy VB. Regulation of nuclear architecture, mechanics, and nucleocytoplasmic shuttling of epigenetic factors by cell geometric constraints. Proc Natl Acad Sci USA 2019; 116: 1320013209.

Bhatti JS, Bhatti GK, Reddy PH. Mitochondrial dysfunction and oxidative stress in metabolic disorders - A step towards mitochondria based therapeutic strategies. Biochimica et Biophysica Acta - Molecular Basis of Disease 2017; $1863: 1066-1077$.

Chang KJ, Redmond SA, Chan JR. Remodeling myelination: Implications for mechanisms of neural plasticity. Nature Neuroscience 2016; 19: 190-197.

Chapman TW, Hill RA. Myelin plasticity in adulthood and aging. Neuroscience Letters 2020; 715: 134645.

Elmore S. Apoptosis: A Review of Programmed Cell Death. Toxicologic Pathology 2007; 35: 495-516.

Hoffmann EK, Lambert IH, Pedersen SF. Physiology of cell volume regulation in vertebrates. Physiological Reviews 2009; 89: 193-277.

Horio T, Murata T, Murata T. The role of dynamic instability in microtubule organization. Vol. 5, Frontiers in Plant Science. Frontiers Media S.A 2014; 5: 511.

Jang J, Quan Z, Yum YJ, Song HS, Paek S, Kang HC. Induced pluripotent stem cells for modeling of pediatric neurological disorders. Biotechnology Journal 2014; 9: 871-891.

Kumar R, Chhikara BS, Gulia K, Chhillar M. Cleaning the molecular machinery of cells via proteostasis, proteolysis and endocytosis selectively, effectively, and precisely: intracellular self-defense and cellular perturbations. Mol Omi 2021; 17: 1128.

Leanza L, Biasutto L, Managò A, Gulbins E, Zoratti M, Szabò I. Intracellular ion channels and cancer. Frontiers in Physiology 2013; 4: 227.

Muñoz-Lasso DC, Romá-Mateo C, Pallardó FV, Gonzalez-Cabo P. Much More Than a Scaffold: Cytoskeletal Proteins in Neurological Disorders. Cells 2020; 9: 358.

Nicolas A, Geiger B, Safran SA. Cell mechanosensitivity controls the anisotropy of focal adhesions. Proc Natl Acad Sci USA 2004; 101: 12520-12525.

Peracchia C. Connexin/innexin channels in cytoplasmic organelles. Are there intracellular gap junctions? a hypothesis! 
Int J Mol Sci 2020; 21: 2163.

Russo R, Ciociaro A, Berliocchi L, Valentina Cassiano MG, Rombolà L, Ragusa S, et al. Implication of limonene and linalyl acetate in cytotoxicity induced by bergamot essential oil in human neuroblastoma cells. Fitoterapia 2013; 89: 48-57.

Tønnesen J. Optogenetic cell control in experimental models of neurological disorders. Behavioural Brain Research $2013 ; 255: 35-43$. 